\title{
Broad Histogram: An Overview
}

\author{
Paulo Murilo Castro de Oliveira \\ Instituto de Física, Universidade Federal Fluminense \\ Av. Litorânea s/n, Boa Viagem, Niterói RJ, Brazil 24210-340 \\ e-mail pmco@if.uff.br
}

Received 4 October, 1999

\begin{abstract}
The Broad Histogram is a method allowing the direct calculation of the energy degeneracy $g(E)$. This quantity is independent of thermodynamic concepts such as thermal equilibrium. It only depends on the distribution of allowed (micro) states along the energy axis, but not on the energy changes between the system and its environment. Once one has obtained $g(E)$, no further effort is needed in order to consider different environment conditions, for instance, different temperatures, for the same system. The method is based on the exact relation between $g(E)$ and the microcanonical averages of certain macroscopic quantities $N^{\mathrm{up}}$ and $N^{\mathrm{dn}}$. For an application to a particular problem, one needs to choose an adequate instrument in order to determine the averages $\left\langle N^{\text {up }}(E)\right\rangle$ and $\left\langle N^{\mathrm{dn}}(E)\right\rangle$, as functions of energy. Replacing the usual fixed-temperature canonical by the fixed-energy microcanonical ensemble, new subtle concepts emerge. The temperature, for instance, is no longer an external parameter controlled by the user. Instead, the microcanonical temperature $T_{m}(E)$ is a function of energy defined from $g(E)$ itself, being thus an internal (environment independent) characteristic of the system. Accordingly, all microcanonical averages are functions of $E$. The present text is an overview of the method. Some features of the microcanonical ensemble are also discussed, as well as some clues towards the definition of efficient Monte Carlo microcanonical sampling rules.
\end{abstract}

\section{Introduction}

The practical interest of equilibrium statistical physics is the determination of the canonical average

$$
<Q>_{T}=\frac{\sum_{S} Q_{S} \exp \left(-E_{S} / T\right)}{\sum_{S} \exp \left(-E_{S} / T\right)}
$$

of a macroscopic quantity $Q$. The system is kept at a fixed temperature $T$, and the Boltzmann constant is set to unity. Both sums run over all possible microstates available for the system, and $E_{S}\left(Q_{S}\right)$ is the value of its energy (quantity $Q$ ) at the particular microstate $S$. The exponential Boltzmann factors take into account the energy exchanges between the system and its environment, in thermodynamic equilibrium.

An alternative is to determine the microcanonical average

$$
\left\langle Q(E)>=\frac{\sum_{S[E]} Q_{S}}{g(E)}\right.
$$

of the same quantity $Q$. In this case, the energy is fixed. Accordingly, the system is restricted to the $g(E)$ degenerate microstates corresponding to the same energy level $E$, and the sum runs over them.

The canonical average (1) can also be expressed as

$$
<Q>_{T}=\frac{\sum_{E}<Q(E)>g(E) \exp (-E / T)}{\sum_{E} g(E) \exp (-E / T)}
$$

where now the sums run over all allowed energy levels E.

Both $g(E)$ and $<Q(E)>$ depend only on the energy spectrum of the system. They do not vary for different environment conditions. For instance, by changing the temperature $T$, the canonical average $<Q>_{T}$ varies, but not the energy functions $g(E)$ and $\langle Q(E)>$ which remain the same. Canonical Monte Carlo simulations are based on equation (1), determining $<Q>_{T}$ : one needs another computer run for each new fixed value of $T$. Instead of this repetitive process, it would be better to determine $g(E)$ and $\langle Q(E)>$ once and forever: canonical averages can thus be calculated from (3), without re-determining $g(E)$ and $\langle Q(E)\rangle$ again for each new temperature.

The Broad Histogram method [1] (hereafter, BHM) is based on the exact relation (4), to be discussed later on. This equation allows one to determine $g(E)$ from the knowledge of the microcanonical averages $\left.<N^{\text {up }}(E)\right\rangle$ and $\left\langle N^{\text {dn }}(E)\right\rangle$ of certain macroscopic quantities: by fixing an energy jump $\Delta E$, the number $N_{S}^{\text {up }}\left(N_{S}^{\text {dn }}\right)$ counts the possible changes one could 
perform on the current microstate $S$, yielding an energy increment (decrement) of $\Delta E$. Adopting a microcanonical computer simulator in order to determine these averages, and also $\langle Q(E)\rangle$, one can calculate canonical averages through (3) without further computer efforts for different temperatures. As the Broad Histogram relation (4) is exact and completely general for any system [2], the only possible source of inaccuracies resides on the particular microcanonical simulator chosen by the user.

BHM does not belong to the class of reweighting methods [3-7]. These are based on the energy probability distribution measured from the actual distribution of visits to each energy level: during the computer simulation, a visit counter $V(E)$ is updated to $V(E)+1$ each time a new microstate is sampled with energy $E$. At the end, the (normalized) histogram $V(E)$ measures the quoted energy probability distribution. It depends on the particular dynamic rule adopted in order to jump from one sampled microstate to the next. One can, for instance, adopt a dynamic rule leading to canonical equilibrium at some fixed temperature $T_{0}$. Then, the resulting distribution can be used in order to infer the behaviour of the same system under another temperature $T[3]$. As canonical probability distributions are very sharply peaked around the average energy, other artificial dynamic rules can also be adopted in order to get broader histograms [4-7], i.e. non-canonical forms of $V(E)$.

BHM is completely distinct from these methods, because it does not extract any information from $V(E)$. The histograms for $N^{\mathrm{up}}(E)$ and $N^{\mathrm{dn}}(E)$ are updated to $N^{\text {up }}(E)+N_{S}^{\text {up }}$ and $N^{\text {dn }}(E)+N_{S}^{\text {dn }}$ each time a new microstate $S$ is sampled with energy $E$. Thus, the information extracted from each sampled state $S$ is not contained in the mere upgrade $V(E) \rightarrow V(E)+1$, but in the macroscopic quantities $N_{S}^{\mathrm{up}}$ and $N_{S}^{\mathrm{dn}}$ carrying a much more detailed description of $S$. In this way, numerical accuracy is much higher within BHM than any other method based on reweighting $V(E)$. Moreover, the larger the system size, the stronger is this advantage, due to the macroscopic character of $N_{S}^{\mathrm{up}}$ and $N_{S}^{\mathrm{dn}}$.

A second feature distinguishing BHM among all other methods is its flexibility concerning the particular way one uses in order to measure the fixed- $E$ averages $\left\langle N^{\text {up }}(E)\right\rangle$ and $\left\langle N^{\text {dn }}(E)\right\rangle$. Any dynamic rule can be adopted in going from the current sampled state to the next, provided it gives the correct microcanonical averages at the end, i.e. a uniform visitation probability to all states belonging to the same energy level $E$. The relative visitation frequency between different energy levels $E$ and $E^{\prime}$ does not matter. Any transition rate definition from level $E$ to $E^{\prime}$ can be chosen, provided it does not introduce any bias inside each energy level, separately. In this way, a more adequate dynamics can be adopted for each different system, still keeping always the full BHM formal- ism and definitions. On the other hand, multicanonical approaches [5-7] are based on a priori unknown transition rates: they are tuned during the simulation in order to get a flat distribution of visits at the end, i.e. a uniform $V(E)$ for which the visiting probability per state is inversely proportional to $g(E)$. By measuring the actually implemented transition rates from $E$ to $E^{\prime}$, which has been tuned during the computer run and must be proportional to $g(E) / g\left(E^{\prime}\right)$, one can finally obtain $g(E)$. Thus, multicanonical approaches are strongly dependent of the particular dynamic rule one adopts. Within BHM, on the other hand, all possible transitions between $E$ and $E^{\prime}$ are exactly taken into account by the quantities $N^{u p}$ and $N^{\text {dn }}$ themselves, not by the particular dynamic transition rates adopted during the computer run.

This text is divided as follows. Section II presents the method, while some available microcanonical simulation approaches are quoted in section III. In section IV, some particularities of the microcanonical ensemble are discussed. Possible improvements in what concerns microcanonical sampling rules are presented in section V. Conclusions are in section VI.

\section{The Method}

Consider a system with many degrees of freedom, denoting by $S$ its current microstate. The replacement of $S$ by another microstate $S^{\prime}$ will be denoted a movement in the space of states. The first concept to be taken into account is the protocol of allowed movements. Each movement $S \rightarrow S^{\prime}$ can be considered allowed or forbiden, only, according to some previously adopted protocol. Nothing to do with the probability of performing or not this movement within some particular dynamic process: BHM is not related to the particular dynamics actually implemented in order to explore the system's space of states. We need to consider the potential movements which could be performed, not the particular path actually followed in the state space, during the actual computer run. Mathematically, the protocol can be defined as a matrix $P\left(S, S^{\prime}\right)$ whose elements are only 1 (allowed movement) or 0 (forbiden). The only restriction BHM needs is that this matrix must be symmetric, i.e. $P\left(S, S^{\prime}\right)=P\left(S^{\prime}, S\right)$, corresponding to microscopic reversibility: if $S \rightarrow S^{\prime}$ is an allowed movement according to the adopted protocol, so is the back movement $S^{\prime} \rightarrow S$. Given a system, one needs first to define this protocol. For an Ising magnet, for instance, one can define that only single-spin flips will be considered. Alternatively, one can accept to flip any set of spins up to a certain number, or to flip clusters of neighbouring spins, or any other protocol. BHM does not depend on which particular protocol is adopted. This free choice can be used in order to improve the efficiency of the method for each particu- 
lar application (besides the already quoted freedom in choosing the simulational dynamics, which has nothing to do with this protocol of virtual movements).

Consider now all the $g(E)$ microstates $S$ belonging to the energy level $E[8]$, and some energy jump $\Delta E>0$ promoting these states to another energy level $E^{\prime}=E+\Delta E$. For each $S$, given the previously adopted protocol, one can count the number $N_{S}^{\text {up }}$ [1] of allowed movements corresponding to this particular energy jump. The total number of allowed movements between energy levels $E$ and $E^{\prime}$, according to the general definition (2) of microcanonical average, is $g(E)<N^{\text {up }}(E)>$. On the other hand, one can consider all the $g\left(E^{\prime}\right)$ microstates $S^{\prime}$ belonging to the energy level $E^{\prime}$ and the same energy jump $-\Delta E<0$, now in the reverse sense. For each $S^{\prime}$ one can count the number $N_{S^{\prime}}^{d n}$ [1] of allowed movements decreasing its energy by $\Delta E$. Due to the above quoted microscopic reversibility, the total number $g\left(E^{\prime}\right)<N^{\mathrm{dn}}\left(E^{\prime}\right)>$ of allowed movements between energy levels $E^{\prime}$ and $E$ is the same as before. Thus, one can write

$$
g(E)<N^{\mathrm{up}}(E)>=g(E+\Delta E)<N^{\mathrm{dn}}(E+\Delta E)>,
$$

which is the fundamental BHM equation introduced in [1]. The method consists in: a) to measure the microcanonical averages $\left\langle N^{\text {up }}(E)\right\rangle,\left\langle N^{\text {dn }}(E)\right\rangle$ corresponding to a fixed energy jump $\Delta E$, and also $\langle Q(E)\rangle$ for the particular quantity $Q$ of interest, storing the results in E-histograms; b) to use (4) in order to determine the function $g(E)$; and $c$ ) to determine the canonical average $\langle Q\rangle_{T}$ from (3), for any temperature. Step a) could be performed by any means. Step b) depends on the previous knowledge of, say, $g(0)$, the ground state degeneracy. However, this common factor would cancel in step c).

There is an alternative formulation of BHM [9], based on a transition matrix approach [10]. Other alternatives can be found in [11-14]. Interesting original analyses were presented in those references. All of them differ from each other only on the particular dynamics adopted in order to measure the BHM averages $\left\langle N^{\text {up }}(E)\right\rangle$ and $\left\langle N^{\text {dn }}(E)\right\rangle$. The common feature is the BHM equation (4). For multiparametric Hamiltonians, the energy $E$ can be replaced by a vector $\left(E_{1}, E_{2} \ldots\right)$ : in this way the whole phase diagram in the multidimensional space of parameters can be obtained from a single computer run [15], representing an enormous speed up.

Besides the freedom of choosing the protocol of allowed movements, the user has also the free choice of the energy jump $\Delta E$. In principle, the same $g(E)$ could be re-determined again for different values of $\Delta E$. Consider, for instance, the uniform Ising ferromagnet on a $L \times L$ square lattice with periodic boundary conditions, and only nearest-neighbour links, for an even
$L>2$. The total energy can be computed as the number of unsatisfied links (pairs of neighbouring spins pointing in opposed senses), i.e. $E=0,4,6,8,10$ $\ldots 2 L^{2}-6,2 L^{2}-4$, and $2 L^{2}$ [16]. Adopting the singlespin-flip protocol of movements, there are two possible values, namely $\Delta E=2$ and $\Delta E=4$, for the energy jumps. Thus, one can determine the same $g(E)$ twice, within BHM. For that, one could store four distinct $E$-histograms: $N^{\text {up }}(\Delta E=4), N^{\text {dn }}(\Delta E=4)$, $N^{\text {up }}(\Delta E=2), N^{\text {dn }}(\Delta E=2)$. The first one corresponds, for each microstate, to the number of spins surrounded by four parallel neighbours, whereas the second to the number of spins surrounded by four neighbours pointing in the opposed sense: together, they determine $g(E)$ through (4), with $\Delta E=4$, from the previous knowledge of $g(0)=2$ and $g(6)=4 L^{2}$. The third and fourth histograms correspond, for each microstate, to the number of spins surrounded by just three or just one parallel neighbours, respectively. Together, they can be used, with $\Delta E=2$, in order to determine $g(E)$ also through (4), from the previous knowledge of $g(4)=2 L^{2}$.

In practice, when Monte Carlo sampling is used as the instrument to measure the microcanonical averages, this freedom on the choice of $\Delta E$ can also be used in order to improve the statistical accuracies $[1,2,17$ 19], by taking all the possible values of $\Delta E$ simultaneously. Provided one has always $\Delta E<<E$ (hereafter the ground state energy will be considered as $E=0$ ), one can store only two E-histograms, with the combinations

$$
N_{S}^{\mathrm{up}}=\sum_{\Delta E}\left[N_{S}^{\mathrm{up}}(\Delta E)\right]^{1 / \Delta E}
$$

and

$$
N_{S}^{\mathrm{dn}}=\sum_{\Delta E}\left[N_{S}^{\mathrm{dn}}(\Delta E)\right]^{1 / \Delta E},
$$

counted at each averaging state. This trick is an approximation very useful in order to save both memory and time. However, it could, in principle, introduce systematic errors, depending on the particular application, and should be avoided in those cases.

\section{Some Microcanonical Simu- lation Approaches}

The averaged value defined by equation (2) refers to all microstates $S[E]$ corresponding to the same energy level $E$, each one being counted just once. For large systems, their number $g(E)$ is normally very large (except, in most cases, near the ground state). In order to obtain a Monte Carlo (random sampling) approximation for $\langle Q(E)>$, one actually averages only over a restricted number of microstates, much smaller than 
$g(E)$. So, these selected averaging states must represent the whole set without any bias, besides the normal statistic fluctuations. The dynamic rule must prescribe exactly the same sampling probability for each state. The appropriate selection of these unbiased averaging states within the same energy level is not an easy task. It depends on the particular system under study, and also on the particular set of allowed movements one adopts. There is no general criterion available in order to assure the uniform sampling probability among fixed- $E$ microstates.

One possible microcanonical simulation strategy is to perform successive random movements, always keeping constant the energy. For instance, the Q2R cellular automaton follows this strategy concerning Ising-like models [20]. Each movement consists in: a) to choose some spin at random; b) to verify whether the energy would remain the same under the flipping of this particular spin; and c) to perform the flip in this case. There is no proof that this strategy is unbiased. Numerical evidences support this possibility, although good averages are obtained only after very, very long transients [21]. On the other hand, these enormous transient times can be avoided [22] by starting the Q2R dynamics from a previously thermalized state, i.e. by running first some canonical steps under a well chosen temperature corresponding to the desired energy. One interpretation of these findings is the following. Combined with the single-spin-flip protocol, the fixed-energy dynamics is a very restrictive strategy in what concerns a fast spread over the whole set of microstates with energy $E$. Indeed, numerical evidences of non ergodicity were found [23]. Nevertheless, either by waiting enormous transient times or by preparing the starting states, Q2R remains a possible choice for microcanonical simulator of Ising systems. However, it will not give good averages at all for very small energies.

An alternative strategy is to relax a little bit the fixed-energy constraint. This idea was introduced [24] by allowing only small energy deviations along the path through the space of states: successive random movements are accepted and performed, provided they keep the energy always inside a small window, i.e. always within a pre-defined set of few adjacent energy levels. Although sampling different energy levels during the same run, the visits to each one are taken into account separately by storing the data in cummulative E-histograms. Even so, each energy level could not be completely free of influences from the neighbouring ones. Nevertheless, this strategy could be a good approximation provided: a) the energy window width $\Delta E$ is very small compared with the energy $E$ itself; and b) the final average $\langle Q(E)>$ is a smooth function of $E$. Again, the method does not work very well for very low energies, where the condition $\Delta E<<E$ cannot be fulfilled. This problem could be partially minimized by adopting some smart tricks [25], although the very low energies would never be well described [18].

A third strategy is to relax completely the fixedenergy constraint, by accepting any energy jump. Now, as $g(E)$ is a fast increasing function of $E$, one is more likely to toss an energy increment than a decrement. The result of accepting any tossed movement would be a fast and irreversible arrival at the maximum entropy region corresponding to infinite temperature, sampling energies only near the maximum of $g(E)$. In order to avoid this, one needs to introduce some acceptance restriction for energy-increasing movements, trying to get a uniform sampling along the whole energy axis. This was first introduced [26] within the distinct context of finding optimal solutions (energy minima) in complex systems. The idea is to divide all the possible movements one could perform, starting from the current microstate, in two classes: increasing or decreasing the energy. First, one of these two classes is randomly tossed. Then, a random movement belonging to the tossed class is performed. This strategy corresponds to an energy random walk, and assures a uniform sampling along the whole energy axis, on average. In spite of this very useful feature in what concerns the search for optimum states in complex systems, this strategy cannot provide correct thermal averages because different energy jumps are mixed together, violating the relative Boltzmann weights between different energy levels. In order to obtain correct thermal averages, one needs to divide the possible movements not in two, but in as many classes as different allowed energy jumps exist: for each fixed positive $\Delta E$, one counts the number of increasing-energy movements $(E \rightarrow E+\Delta E)$ and the number of decreasing-energy ones $(E \rightarrow E-\Delta E)$, the same $\Delta E$ for both, in order to accept or not the currently tossed movement. In other words, one needs to consider precisely the $\Delta E$-dependent BHM quantities $N_{S}^{\mathrm{up}}$ and $N_{S}^{\mathrm{dn}}$ defined in [1].

Many variants of this energy random walk dynamics can be defined, the best one [27] being a direct consequence of the BHM equation (4) itself, as follows. In order to obtain a flat distribution of visits along the energy axis, one needs: a) to toss a random movement starting from the current microstate with energy $E$; b) to perform it whenever the energy decreases; and $c$ ) to perform it only with probability $g(E) / g(E+\Delta E)$, whenever the energy increases $(\Delta E>0$ being the increment). From the exact relation (4), once one has some previous estimate of the energy functions $\left\langle N^{\mathrm{up}}(E)>0\right.$ and $<N^{\mathrm{dn}}(E)>_{0}$, this probability is also equal to

$$
p_{\text {up }}=\frac{<N^{\mathrm{dn}}(E+\Delta E)>_{0}}{<N^{\text {up }}(E)>_{0}} .
$$

The dynamic rule proposed in [27] is then based on a two-step computer simulation. First, one obtains an estimate of $<N^{\mathrm{up}}(E)>_{0}$ and $<N^{\mathrm{dn}}(E)>_{0}$, by any means. Then, using this first estimate in a further, independent computer run, one performs the 
above defined dynamics, energy-increasing movements $(E \rightarrow E+\Delta E$ ) being accepted only according to the probability (6). From this second run, one measures accurate (according to [27]) averages $\left\langle N^{\text {up }}(E)\right\rangle$ and $<N^{\text {dn }}(E)>$ from which $g(E)$ can be finally obtained from the BHM relation (4).

This flat histogram dynamic recipe was proposed [27] in order to solve some numerical deviations observed in previous versions which could be viewed as approximations to it. As discussed in section IV, unfortunately things are not so easy. These problems are not merely related to particular dynamic approximate recipes, but to another characteristic of the system itself: the discreteness of the energy spectrum. All fundamental concepts leading to the microcanonical ensemble are based on the supposition that all energy changes $\Delta E$ are much smaller than the current energy $E$. In other words, microcanonical ensemble is defined by disregarding the energy spectrum discreteness. That is why conceptual problems appear: a) at very low energies, for any system; b) at any energy, for tiny systems. A better understanding of these subtle concepts cannot be obtained by simple improvements of the dynamic recipe: a new conceptual framework allowing to treat also discrete spectra is needed (and lacking).

On the other hand, excepting for the two situations quoted in the above paragraph, the various approximations to the acceptance rates (6) are not so bad as supposed in [27], according to the evidences shown also in sections IV and V. Thus, let's quote these approximations which make things easier. First, instead of performing two computer runs in order to obtain the transition rates (6) from the first and averages from the second, one can perform a single one gradually accumulating $\left\langle N^{\text {up }}(E)>\right.$ and $\left\langle N^{\mathrm{dn}}(E)\right\rangle$ in E-histograms. At each step, in order to decide whether the currently tossed movement must be performed or not, one uses the already accumulated values themselves, by reading both the numerator and the denominator of (6) from the corresponding histograms at the proper energy channels $E$ and $E+\Delta E$. Actually, this trick was already introduced (and used) in the original publication [1]. This approximation will be denoted by A1. It follows the same lines of real-time-defined transition rates of the multicanonical sampling methods [5-7]. A further approximation, hereafter called A2, consists in ignoring the $\Delta E$ appearing in the numerator of (6), reading both the numerator and the denominator from the same energy channel $E$. This saves two real division operations by the current number of visits $V(E)$ and $V(E+\Delta E)$. The third additional approximation consists in taking together all possible energy jumps $\Delta E$ through equations (5a) and (5b), hereafter called A3. This saves computer memory, because only a pair of histograms, one for $\left\langle N^{\text {up }}(E)\right\rangle$ and the other for $<N^{\mathrm{dn}}(E)>$ are needed, instead of a pair for each different possible value of $\Delta E$. Also, this approxima- tion saves time because the statistic fluctuations are not spread over many histograms, the whole available data being superimposed in only two.

A further yet approximation is simply to replace the numerator and the denominator of (6) by the current values $N_{S}^{\text {up }}$ and $N_{S}^{\text {dn }}$ corresponding to the current microstate $S$, instead of reading previously averaged values. In theory, for large systems, this replacement could be justified if $N^{\text {up }}$ and $N^{\text {dn }}$ are shown to be self averaging quantities, in spite of the further fluctuations it introduces. However, contrary to the cases A1, A2 and A3 (described in the last paragraph and actually tested $[1,2,17-19])$, this procedure does not save any computer time or memory, being useless in practice.

On the other hand, the procedure [28] of counting $N_{S}^{\text {up }}$ and $N_{S}^{\text {dn }}$ at the current microstate $S$, without classifying them according to different values of $\Delta E$, is no longer an approximation: it is wrong in what concerns the measurement of averages, because the relative Boltzmann probability for different energies would be violated. It corresponds to the mistake of missing the exponent $1 / \Delta E$ in equations (5a) and (5b). Only when applied to the completely different context of finding optimal solutions in complex systems [26], without performing averages, this procedure is justifiable.

The original multicanonical approach [5] can be reformulated according to the entropic sampling dynamics [6]. The degeneracy function $g(E)$ is gradually constructed during the computer run. It is based on an acceptance probability $g(E) / g\left(E^{\prime}\right)$ for each new tossed movement from the current energy $E$ to another value $E^{\prime}$. After some steps, the whole function $g(E)$ is updated according to the new visit trials, and so on. However, this method is yet based exclusively on the histogram for $V(E)$. Another possibility [29] is to adopt exactly this same dynamic rule in order to sample the averaging states, measuring also the microcanonical averages $\left\langle N^{\text {up }}(E)\right\rangle$ and $\left\langle N^{\text {dn }}(E)\right\rangle$ during the computer run. At the end, $g(E)$ is obtained through the BHM equation (4), instead of the values updated during the simulation: the results [29], of course, are much more accurate. Note that, in this case, exactly the same microstates are visited, i.e. the same Markovian chain of averaging states. In this way, the better BHM performance is explicitly shown to be a consequence of the more detailed description of each averaging state, compared with reweighting, multicanonical approaches.

Uniform probability distributions along the energy axis may be not the best strategy. Being independent of the particular simulation dynamics, BHM allows one to get better sampling statistics in some energy regions. In [19] the Creutz dynamics [24,18] is combined with the energy random walk [1]. First, one chooses a window corresponding to few adjacent energy levels. Then, the energy random walk dynamics is applied inside this window up to a certain predefined number of averaging states were sampled. After that, the window is moved 
one energy level up, by including the next allowed energy level at right and removing the leftmost. Then, the same procedure is repeated inside this new window up to a slightly larger number of averaging states, and so on. Reaching the critical region, this number is kept fixed at its maximum value, before to go down again for energies above the critical region. In this way, BHM allows the user to design the profile of visits along the energy axis, according to the numerical accuracy needed within each region.

\section{Canonical $\times$ Microcanonical Simulations}

The equivalence between the various thermodynamic ensembles (canonical and microcanonical, for instance) is a widespread belief. However, this is true only in the so-called thermodynamic limit $N \rightarrow \infty$, where $N$ is the number of components forming the system under study. For finite systems, and thus for any computer simulational approach, the lack of this equivalence [30] poses serious conceptual as well as practical problems.

Canonical computer simulation approaches were very well developed since the pioneering work [31], half a century ago. By following some precise recipes (random movements transforming the current microstate into the next one) a Markovian chain of averaging states is obtained, from which thermodynamic canonical averages are calculated. As a consequence of this conceptual development, particular recipes were shown to provide unbiased canonical equilibrium. On the other hand, microcanonical simulation has never attracted the attention of researchers during this same half century (with some few exceptions). That is why some fundamental concepts concerning this subject are misunderstood in the literature.

The direct way to construct a fixed- $E$, microcanonical simulator would be to accept a new randomly tossed movement only if it does not change the energy. However, this constraint could introduce non-ergodicity problems, depending on the particular set of movements one adopts. For the Ising model, for instance, this problem seems to occur by adopting only one-spin flips [21$23]$. In order to minimize it, one needs to allow morethan-one-spin flips [32]. However, by flipping only two spins far away from each other, after each whole-lattice one-spin-flip sweep, the magnetic order is destroyed below the critical energy [32]. Thus, the strictly fixed energy approach is problematic, and the alternative is to relax it, allowing some energy changes. However, this also poses troubles, once the equilibrium features (magnetization, correlations, etc.) of the system at some energy level $E$ are not exactly the same at another level $E^{\prime}$. By travelling from $E$ to $E^{\prime}$ without time enough for equilibration, one could introduce biases from $E_{-}$ states into $E^{\prime}$ averages: the multiple-energy dynamics could distort the strictly uniform probability distribution inside each energy level. In short, the construction of a good microcanonical simulator is not a simple subject.

If you have not enough conceptual understanding about some particular subject, a good idea is to resort to another similar subject for which your conceptual understanding is already firmly stablished. Let's define a particular microcanonical simulator by using well stablished canonical rules, for which the temperature $T$ is fixed since beginning, being an external control parameter. The canonical average of any macroscopic quantity $Q$ becomes a function of $T$, as $\langle Q\rangle_{T}$ in equations (1) or (3). Let's consider some finite system with $N$ components, and its average energy $<E\rangle_{T}$. Although the energy spectrum is discrete [8], $\langle E\rangle_{T}$ is a continuous function of $T$ (except for a possible isolated temperature where a first order transition may occur). Thus, one can tune the value of $T$ in order to have $<E\rangle_{T}$ coincident with some previously chosen energy level $E$ : let's call this tuned temperature $T(E)$. Then, a correct microcanonical simulator recipe is: a) to run some of the many known canonical recipes with fixed temperature $T(E)$; b) to measure the quantity $Q_{S}$ of interest, for each averaging state $S$ whose energy is $E$; c) to accumulate $Q_{S}$ as well as the number of visits to the particular energy level $E$, during the run; and d) to calculate the microcanonical average $\langle Q(E)\rangle$ at the end, by dividing the accumulated sum of $Q_{S}$ by the number of visits to level $E$. This recipe, of course, may not be very efficient, once one will visit many energy levels, others than the previously fixed value $E$, storing data concerning only this particular level. Also, the precise temperature $T(E)$ must be known a priori, and perhaps this knowledge could be achieved only by performing some previous runs. Moreover, the whole process must be repeated for each different energy level. Nevertheless, this recipe is perfectly correct, and will be the basis for our reasonings hereafter.

Table I shows the exact data for a square $4 \times 4$ lattice Ising ferromagnet, obtained by direct counting all the $2^{16}=65.536$ possible microstates. The adopted protocol of (virtual) movements is the set of all possible one-spin flips. From the first two columns, the exact temperatures $T(E)$ can be obtained for each energy level $E$, by analytically calculating $\left\langle E>_{T}\right.$ as a function of $T$ from equation (3), and then imposing $<E\rangle_{T}=E$. Table II shows the data obtained from canonical simulation with fixed $T(8)=3.02866216$ (in the usual units where the energy corresponding to each bond is $\pm J$, instead of 0 or 1 ), $10^{8}$ whole lattice sweeps, and 32 independent samples. Thus, the total number of averaging states is $3.2 \times 10^{9}$. For all averaged values displayed, statistical fluctuations occur at most on the two rightmost digits. The second column shows the actual number $V(E)$ of visits to each energy level. The expected statistical relative deviations are, thus, of the 
order of $V(E)^{-1 / 2}$ in each case, in agreement with the ones actually obtained (roughly 1 part in $10^{4}$ ). Within this statistical accuracy, the coincidence between the first line in Table II and the corresponding exact values for $E=8$, in Table I, is a further evidence confirming that the microcanonical simulator defined in the above paragraph is indeed correct: it does not introduce any bias besides the normal statistical numerical fluctuations.

Tables III to $\mathrm{V}$ show data obtained from canonical simulations with fixed temperatures $T(10)=$ $3.57199419, T(12)=4.66862103$ and $T(14)=$ 8.33883787 , respectively. These values were tuned in order to give the exact average energies $\langle E\rangle_{T}=10$, 12 and 14 , respectively. Note again the coincidence of the second line in Table III, the third line in Table IV, as well as the fourth line in Table $\mathrm{V}$ with the exact values presented in Table I, within the numerical accuracy.

Level $E=16$ is just the center of the energy spectrum, corresponding to $T(16)=\infty$. In order to simulate this situation, we adopted a very high temperature, namely $T=100$, in Table VI. Its fifth line is supposed to be compared with the corresponding exact values in Table I.

The other lines in Tables II to VI also coincide with the corresponding exact values in Table I, within the numerical accuracy. However, these further coincidences are not completely trustable, because Table II, for instance, was obtained by fixing the simulational temperature $T(8)$ tuned in order to give the average energy $<E>_{T}=8$. Thus, this Table II is out of tune for the other energy levels $E \neq 8$. Accordingly, Tables III, IV, $\mathrm{V}$ and VI are out of tune for energies $E \neq 10, E \neq 12, E \neq 14$ and $E \neq 16$, respectively. Indeed, considering for instance the simulations at fixed $T(E=14)$, the relative deviation obtained for $N^{\mathrm{dn}}(E=8)$ with $\Delta E=2$ and 4 are respectively 14 or 18 times larger than the expected 1 part in $10^{4}$. In principle, only data obtained from canonical simulations performed at the right temperature $T(E)$ (i.e. the one for which $\langle E\rangle_{T}=E$ ) could be taken as microcanonical averages for this particular energy level $E$. However, the systematic deviations induced by taking also data corresponding to neighboring energy levels others than $E$ seem to be very small. In particular, for larger systems, and near the critical region, justifying the simple approach of adding histograms obtained at different temperatures $[17,9]$ : in those cases, the temperature is a slowly varying function of $E(\mathrm{~d} T / \mathrm{d} E=0$ at the critical point, in the thermodynamic limit).

$\begin{array}{rrccccc}E & g(E) & \begin{array}{c}\langle\mathrm{up} \\ (\Delta E)\rangle\end{array} \quad & \left\langle N^{\mathrm{up}}(E)\right\rangle & \left\langle N^{\mathrm{tie}}(E)\right\rangle & \left\langle N^{\mathrm{dn}}(E)\right\rangle & \left\langle N^{\mathrm{dn}}(E)\right\rangle \\ & & (\Delta E=2) & (\Delta E=0) & (\Delta E=2) & (\Delta E=4) \\ 0 & 2 & 16 & 0 & 0 & 0 & 0 \\ 4 & 32 & 11 & 4 & 0 & 0 & 1 \\ 6 & 64 & 8 & 6 & 0 & 2 & 0 \\ 8 & 424 & 6.11321 & 6.33962 & 1.81132 & 0.90566 & 0.83019 \\ 10 & 1728 & 3.55556 & 7.03704 & 3.55556 & 1.55556 & 0.29630 \\ 12 & 6688 & 2.31101 & 5.97129 & 5.51196 & 1.81818 & 0.38756 \\ 14 & 13568 & 1.13208 & 5.58491 & 5.88679 & 2.94340 & 0.45283 \\ 16 & 20524 & 0.75307 & 3.69207 & 7.10973 & 3.69207 & 0.75307\end{array}$

Table I Exact data concerning the $4 \times 4$ square lattice Ising ferromagnet. The second half of the spectrum $(E=18 \ldots 32)$ is symmetric the to first half $(E=14 \ldots 0) . N^{\text {tie }}$ is the number of potential movements (one-spin flips) keeping the energy unchanged.

$\begin{array}{rcccccc}E & V(E) & \left\langle N^{\mathrm{up}}(E)\right\rangle & \left\langle N^{\mathrm{up}}(E)\right\rangle & \left\langle N^{\text {tie }}(E)\right\rangle & \left\langle N^{\mathrm{dn}}(E)\right\rangle & \left\langle N^{\mathrm{dn}}(E)\right\rangle \\ & \times 10^{8} & (\Delta E=4) & (\Delta E=2) & (\Delta E=0) & (\Delta E=2) & (\Delta E=4) \\ 8 & 5.62 & 6.1145 & 6.3376 & 1.81155 & 0.90606 & 0.83028 \\ 10 & 6.11 & 3.5565 & 7.0366 & 3.5540 & 1.55630 & 0.29665 \\ 12 & 6.32 & 2.3111 & 5.9711 & 5.5119 & 1.81843 & 0.38744 \\ 14 & 3.42 & 1.13151 & 5.5851 & 5.8879 & 2.9429 & 0.45259 \\ 16 & 1.38 & \mathbf{0 . 7 5 3 0 2} & 3.6916 & 7.1109 & 3.6913 & 0.75315\end{array}$

Table II Canonical simulation for the $4 \times 4$ square lattice Ising ferromagnet, with a fixed temperature $T(8)=3.02866216$ for which the exact energy average is $\langle E\rangle_{T}=8$. For all averaged values, statistical fluctuations fall on the two last digits, at most. 


$\begin{array}{rcccccc}E & V(E) & \left\langle N^{\mathrm{up}}(E)\right\rangle & \left\langle N^{\mathrm{up}}(E)\right\rangle & \left\langle N^{\mathrm{tie}}(E)\right\rangle & \left\langle N^{\mathrm{dn}}(E)\right\rangle & \left\langle N^{\mathrm{dn}}(E)\right\rangle \\ & \times 10^{8} & (\Delta E=4) & (\Delta E=2) & (\Delta E=0) & (\Delta E=2) & (\Delta E=4) \\ 8 & 4.68 & 6.1123 & 6.3410 & 1.81107 & 0.90577 & 0.82989 \\ 10 & 6.22 & 3.5559 & 7.0367 & 3.5552 & 1.55578 & 0.29639 \\ 12 & 7.85 & 2.3113 & 5.9713 & 5.5115 & 1.81836 & 0.38771 \\ 14 & 5.20 & 1.13148 & 5.5858 & 5.8870 & 2.9426 & 0.45307 \\ 16 & 2.57 & 0.75274 & 3.6914 & 7.1116 & 3.6917 & 0.75257\end{array}$

Table III The same as Table II, now with a fixed temperature $T(10)=3.57199419$ for which the exact energy average is $\langle E\rangle_{T}=10$.

$\begin{array}{rcccccc}E & V(E) & \left\langle N^{\mathrm{up}}(E)\right\rangle & \left\langle N^{\mathrm{up}}(E)\right\rangle & \left\langle N^{\text {tie }}(E)\right\rangle & \left\langle N^{\mathrm{dn}}(E)\right\rangle & \left\langle N^{\mathrm{dn}}(E)\right\rangle \\ & \times 10^{8} & (\Delta E=4) & (\Delta E=2) & (\Delta E=0) & (\Delta E=2) & (\Delta E=4) \\ 8 & 3.08 & 6.1122 & 6.3404 & 1.8116 & 0.90667 & 0.82909 \\ 10 & 5.34 & 3.5550 & 7.0372 & 3.5552 & 1.55529 & 0.29597 \\ 12 & 8.78 & 2.31065 & 5.9707 & 5.5138 & 1.81777 & 0.38711 \\ 14 & 7.56 & 1.13206 & 5.5846 & 5.8872 & 2.9436 & 0.45255 \\ 16 & 4.85 & 0.75310 & 3.6923 & 7.1091 & 3.6924 & 0.75303\end{array}$

Table IV The same again, now with a fixed temperature $T(12)=4.66862103$ for which the exact energy average is $\langle E\rangle_{T}=12$.

$\begin{array}{rcccccc}E & V(E) & \left\langle N^{\mathrm{up}}(E)\right\rangle & \left\langle N^{\mathrm{up}}(E)\right\rangle & \left\langle N^{\mathrm{tie}}(E)\right\rangle & \left\langle N^{\mathrm{dn}}(E)\right\rangle & \left\langle N^{\mathrm{dn}}(E)\right\rangle \\ & \times 10^{8} & (\Delta E=4) & (\Delta E=2) & (\Delta E=0) & (\Delta E=2) & (\Delta E=4) \\ 8 & 1.30 & 6.1137 & 6.3404 & 1.8098 & 0.90444 & 0.83169 \\ 10 & 3.27 & 3.5550 & 7.0379 & 3.5554 & 1.55540 & 0.29628 \\ 12 & 7.84 & 2.31063 & 5.9716 & 5.5123 & 1.81816 & 0.38735 \\ 14 & 9.83 & 1.13243 & 5.5845 & 5.8866 & 2.9436 & 0.45291 \\ 16 & 9.20 & 0.75357 & 3.6916 & 7.1094 & 3.6920 & 0.75339\end{array}$

Table $\mathrm{V}$ The same once more, now with a fixed temperature $T(14)=8.33883787$ for which the exact energy average is $\langle E\rangle_{T}=14$.

$\begin{array}{rrccccc}E & V(E) & \left\langle N^{\mathrm{up}}(E)\right\rangle & \left\langle N^{\mathrm{up}}(E)\right\rangle & \left\langle N^{\mathrm{tie}}(E)\right\rangle & \left\langle N^{\mathrm{dn}}(E)\right\rangle & \left\langle N^{\mathrm{dn}}(E)\right\rangle \\ & \times 10^{8} & (\Delta E=4) & (\Delta E=2) & (\Delta E=0) & (\Delta E=2) & (\Delta E=4) \\ 8 & 0.35 & 6.1132 & 6.3405 & 1.8106 & 0.90464 & 0.83108 \\ 10 & 1.40 & 3.5560 & 7.0366 & 3.5552 & 1.55577 & 0.29642 \\ 12 & 5.22 & 2.31103 & 5.9717 & 5.5112 & 1.81838 & 0.38769 \\ 14 & 10.17 & 1.13244 & 5.5847 & 5.8863 & 2.9436 & 0.45298 \\ 16 & 14.77 & 0.75265 & 3.6922 & 7.1102 & 3.6922 & 0.75266\end{array}$

Table VI Again, now with a fixed temperature $T=100$, mimicking $T(16)=\infty$.

In the thermodynamic limit, the canonical temperature can be obtained by the statistical definition

$$
\frac{1}{T}=\frac{\mathrm{d}}{\mathrm{d} \epsilon} \lim _{N \rightarrow \infty} \frac{\ln g(N \epsilon)}{N}
$$

where $\epsilon=E / N$ is the energy density, and coincides perfectly with the value $T(E)$ quoted before, for which $<E>_{T}=E$. However, for finite systems, both the thermodynamic limit $N \rightarrow \infty$ as well as the derivative limit $\Delta \epsilon=\Delta E / N \rightarrow 0$ cannot be performed. A palia- tive procedure is simply to forget them, transforming equation (7) in

$$
\frac{1}{T_{m}(E)}=\frac{\ln g(E+\Delta E)-\ln g(E)}{\Delta E},
$$

where the subscript $m$ means "microcanonical temperature" defined just now for finite systems, and $\Delta E$ is the energy gap between level $E$ and some other level above it. Of course, in principle, $T_{m}(E)$ also depends on $\Delta E$, which indicates a first difference between this and the 
true canonical temperature. Moreover, contrary to the real, canonical temperature $T(E)$, this $T_{m}(E)$ is not an external parameter depending on the system's environment and equilibrium conditions: it is simply an alternative formulation for the energy spectrum $g(E)$ itself, being thus environment-independent. Note also that $T_{m}(E)$ is defined only at the allowed energies belonging to the discrete spectrum, whereas the real temperature $T(E)$ (the one for which $\langle E\rangle_{T}=E$ ) can be defined for any value $E$, continuously. An alternative formula is

$$
\frac{1}{T_{m}(E+\Delta E / 2)}=\frac{\ln g(E+\Delta E)-\ln g(E)}{\Delta E} .
$$

The most famous canonical recipe [31] is: a) to toss some random movement, starting from the current state; b) to calculate the energy variation $\Delta E$ this movement would promote if actually implemented; c) to perform the movement, whenever $\Delta E \leq 0$, counting one more step; and $d$ ) to perform the movement only with the Boltzmann acceptance probability $\exp (-\Delta E / T)$, if $\Delta E>0$, counting one step anyway. Normally, one takes a new averaging state after $N$ successive steps (one MC step). Let's stress that, here, the temperature $T$ is the real, canonical one: in order to use this recipe to measure microcanonical averages at some fixed energy $E$, one must take $T=T(E)$, the temperature for which $\langle E\rangle_{T}=E$. Instead, by taking $T=T_{m}(E)$ in equation (8a), it is an easy exercise to show that the Boltzmann acceptance probability $\exp (-\Delta E / T)$ would be equal to $g(E) / g(E+\Delta E)$. But this is just the acceptance probability adopted within the flat histogram dynamics [27].

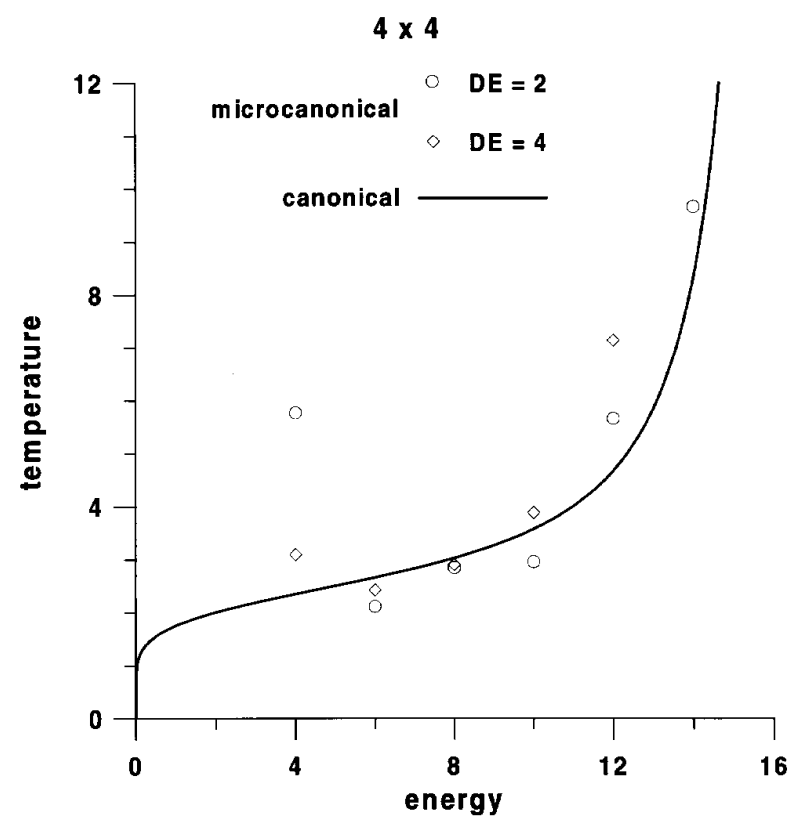

Figure 1. Exact canonical temperature as a function of the energy (continuous line), for a $4 \times 4$ square lattice Ising ferromagnet. Symbols correspond to the microcanonical version of the temperature, equation (8a), with energy gaps $\Delta E=2$ (circles) or 4 (diamonds).

Fig. 1 shows $T_{m}(E)$ calculated from equation (8a), for a $4 \times 4$ square Ising ferromagnet. The exact values of $g(E)$ were used. The open circles correspond to $\Delta E=2$, and the diamonds to $\Delta E=4$. The continuous line is the exact $T(E)$, also calculated from the exact values of $g(E)$. The deviations between $T_{m}(E)$ and $T(E)$ are very strong. Note that there is no approximation at all, neither in $T_{m}(E)$ nor in $T(E)$. The deviations represent true differences between canonical and microcanonical ensembles, which are indeed very strong for this tiny system. Obviously, the condition $\Delta E<<E$ is not fulfilled, and the discreteness is inevitable along the whole energy spectrum.

Fig. 2 reports the same data as Fig. 1, with the same symbols, now for a $32 \times 32$ lattice, and using equation (8b). The exact values for $g(E)$ were taken from [33]. The same strong deviations occur again, but now only at the very beginning of the energy spectrum, where the condition $\Delta E<<E$ does not hold, as can be seen in the upper inset. However, near the critical region, the deviations become much smaller, as can be seen in the lower inset where the vertical scale is 100 times finer than the upper one. For $E>64$, the energy spectrum discreteness can be neglected within a very good approximation, even with $N=1024$ being still very far from the thermodynamic limit in equation (7). The larger the system size, the better becomes the situation, because the relation $\Delta E<<E$ becomes more and more fulfilled. However, at the very beginning of the spectrum this relation will never be fulfilled, even for large systems.

Both limits in equation (7), namely the thermodynamic one $N \rightarrow \infty$ and $\Delta E \rightarrow 0$ corresponding to the energy derivative, were neglected in equation (8a). However, only the latter seems to have disastrous consequences when one uses $T_{m}(E)$ as an approximation for $T(E)$. Indeed, even for very small systems like the $32 \times 32$ square lattice (not so tiny as $4 \times 4$ ), the deviations are very small, provided the condition $\Delta E<<E$ holds. In other words, the deviations between the microcanonical temperature $T_{m}(E)$ and the true canonical value $T(E)$ comes almost exclusively from the breakdown of the condition $\Delta E<<E$, not from finite size effects. Even in the thermodynamic limit, $T_{m}(E)$ will differ from $T(E)$ near the ground state, due to the discretness of the energy spectrum. On the other hand, to take $T_{m}(E)$ instead of $T(E)$ is a very, very good approximation far from the ground state: we have seen before that microcanonical averages obtained from canonical simulations are not sensitive to temperatures out of tune for a given energy, moreover when the deviations are of the order of that shown in the lower inset of Fig. 2. 


\section{$32 \times 32$}

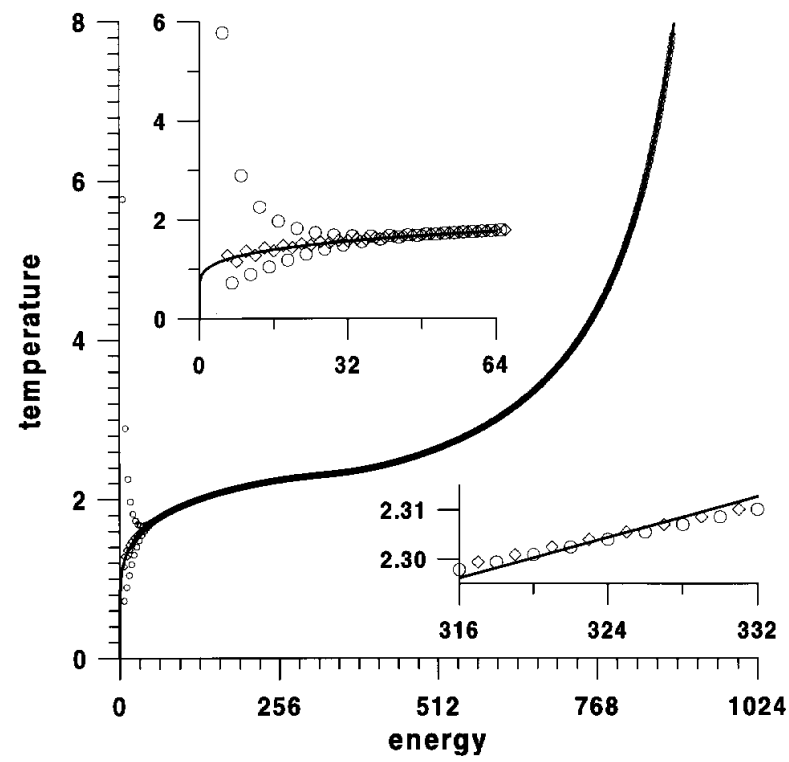

Figure 2. The same as figure 1, now for a $32 \times 32$ lattice, with the same symbols, and equation $(8 \mathrm{~b})$. Note that the deviations between the true canonical temperatures and the microcanonical versions are now restricted to the very beginning of the energy spectrum (upper inset). At the critical region these deviations are much smaller (lower inset, with a 100 times finer scale). The deviations become smaller yet for larger systems.

\section{exact $\circ 32 \times 32$}

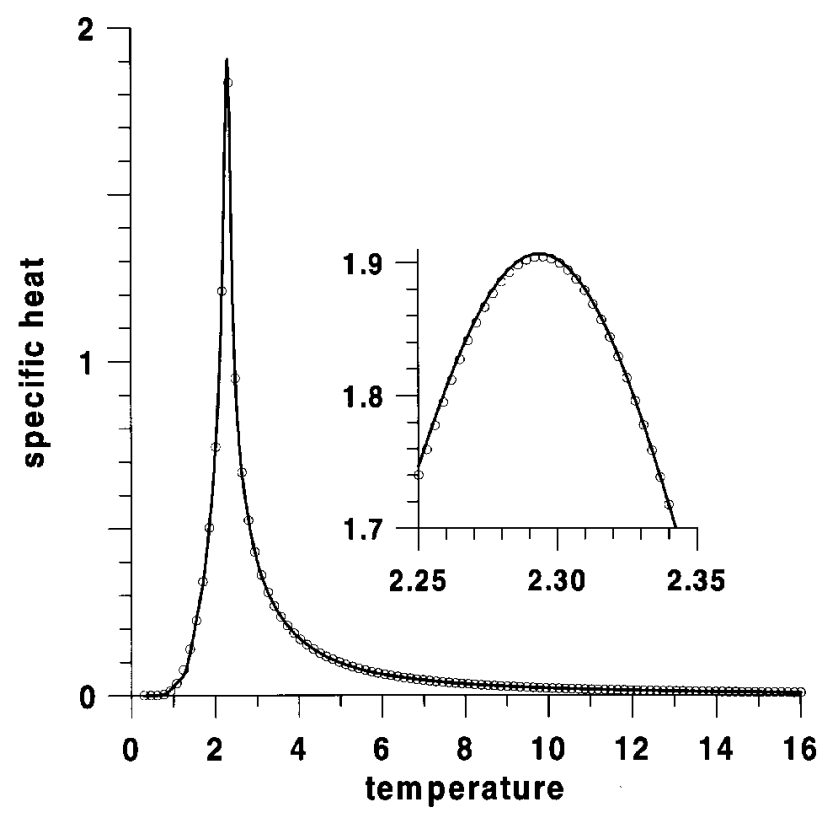

Figure 3. Specific heat for the $32 \times 32$ lattice Ising ferromagnet, adopting the random walk dynamics within restricted energy windows [19]. Circles show the exact values [33].
Tables II to VI present good numerical accuracies not only for the tuned temperatures, but also when out-of-tune values for $T$ were considered. Moreover, based on the reasonings above, this feature will be even improved for larger and larger systems. The imediate consequence is the possibility of adding different histograms for $\left\langle N^{\mathrm{up}}(E)\right\rangle$ and $\left\langle N^{\mathrm{dn}}(E)\right\rangle$, obtained from distinct canonical simulations with different temperatures, as already tested in $[17,9]$. Of course, this approach is much more efficient than to take a different canonical simulation with fixed temperature $T(E)$ for each different energy level $E$, without superimposing the histograms. However, it still needs many computer runs, one for each fixed temperature.

In order to improve even more the efficiency, one can try the following strategy. First, one defines the Boltzmann acceptance probability $\exp [-\Delta E / T(E)]$ for each energy level $E$ and each possible energy jump $\Delta E$. Note that this is not the same as canonical simulations where the acceptance probability $\exp (-\Delta E / T)$ depends only on $\Delta E$ but not on $E$. Then, by following this non-canonical, $E$-dependent acceptance probability, one runs a single computer simulation, visiting the whole energy axis, accumulating the histograms for $\left\langle N^{\text {up }}(E)\right\rangle$ and $\left\langle N^{\text {dn }}(E)\right\rangle$. This approach is similar to the flat histogram dynamics [27], using the true temperature $T(E)$ instead of the microcanonical value $T_{m}(E)$. Table VII shows the results for the same tiny system already considered before. Surprisingly, strong deviations appear. In order to analyse the source of these deviations, let's introduce another very similar alternative, adopting a different acceptance probability $\exp [-\Delta E / T(E+\Delta E / 2)]$. The canonical Boltzmann probability is taken at the center $E+\Delta E / 2$ of the interval correponding to the energy jump from $E$ to $E+\Delta E$, not at the current energy $E$. This symmetrization trick is supposed to diminish the numerical deviations due to impossibility of performing the limit $\Delta E \rightarrow 0$, i.e. the derivative in equation ( 7$)$, for this tiny system. The results are shown in Table VIII, in complete agreement with the exact results, Table I. Thus, the source of the deviations in Table VII is the lack of the condition $\Delta E<<E$. By taking into account both the current energy level $E$ as well as the (wouldbe) next energy $E+\Delta E$ in a symmetric way, the numerical deviations were eliminated. Of course, for larger systems and far from the ground state, where the condition $\Delta E<<E$ holds, the differences between Tables VII and VIII would also disappear.

The flat histogram dynamics uses another acceptance probability, namely

$$
\exp \left[-\Delta E / T_{m}(E)\right]=\frac{g(E)}{g(E+\Delta E)}=\frac{<N^{\mathrm{dn}}(E+\Delta E)>}{\left\langle N^{\mathrm{up}}(E)\right\rangle}
$$


where $E$ and $E+\Delta E$ also play a symmetric role. Simulational results are shown in Table IX, where the exact values for $g(E)$ (or, alternatively, $\left\langle N_{\text {up }}(E)\right\rangle$ and $\left\langle N_{\mathrm{dn}}(E)\right\rangle$ ) were adopted in order to determine the acceptance probabilities (9). The results are again coincident with the expected ones, Table I [34]. On the other hand, if one tries to break the quoted symmetry, ignoring $\Delta E$ in the numerator of the right-hand-side term in (9), i.e. approximation A2, numerical deviations similar to Table VII would appear, for this tiny system.

$\begin{array}{rcccccc}E & V(E) & \left\langle N^{\mathrm{up}}(E)\right\rangle & \left\langle N^{\mathrm{up}}(E)\right\rangle & \left\langle N^{\mathrm{tie}}(E)\right\rangle & \left\langle N^{\mathrm{dn}}(E)\right\rangle & \left\langle N^{\mathrm{dn}}(E)\right\rangle \\ & \times 10^{8} & (\Delta E=4) & (\Delta E=2) & (\Delta E=0) & (\Delta E=2) & (\Delta E=4) \\ 8 & 3.91 & 6.1075 & 6.3168 & 1.8413 & 0.93716 & 0.79731 \\ 10 & 4.11 & 3.5647 & 7.0105 & 3.5752 & 1.55947 & 0.29019 \\ 12 & 4.96 & 2.32037 & 5.9336 & 5.5420 & 1.83372 & 0.37031 \\ 14 & 3.99 & 1.13602 & 5.5671 & 5.8935 & 2.9675 & 0.43584 \\ 16 & 3.55 & 0.73739 & 3.6917 & 7.1232 & 3.7290 & 0.71873\end{array}$

Table VII Random walk, pseudo-canonical simulation with asymmetric $E$-dependent Boltzmann acceptance probability $\exp [-\Delta E / T(E)]$, to be compared with the exact values in Table I.

$\begin{array}{rcccccc}E & V(E) & \left\langle N^{\mathrm{up}}(E)\right\rangle & \left\langle N^{\mathrm{up}}(E)\right\rangle & \left\langle N^{\mathrm{tie}}(E)\right\rangle & \left\langle N^{\mathrm{dn}}(E)\right\rangle & \left\langle N^{\mathrm{dn}}(E)\right\rangle \\ & \times 10^{8} & (\Delta E=4) & (\Delta E=2) & (\Delta E=0) & (\Delta E=2) & (\Delta E=4) \\ 8 & 3.25 & 6.1134 & 6.3392 & 1.81132 & 0.90608 & 0.82998 \\ 10 & 3.87 & 3.5571 & 7.0343 & 3.5561 & 1.55637 & 0.29609 \\ 12 & 5.48 & 2.31315 & 5.9677 & 5.5123 & 1.81968 & 0.38715 \\ 14 & 5.57 & 1.13256 & 5.5833 & 5.8871 & 2.9458 & 0.45130 \\ 16 & 6.56 & 0.75220 & 3.6918 & 7.1109 & 3.6939 & 0.75118\end{array}$

Table VIII Random walk, pseudo-canonical simulation with symmetric $E$-dependent Boltzmann acceptance probability $\exp [-\Delta E / T(E+\Delta E / 2)]$, to be compared with the exact values in Table I.

As one does not know a priori $g(E)$ (or, alternatively, $<N_{\text {up }}(E)>$ and $<N_{\text {dn }}(E)>$ ), one can use some previous estimates $<N_{\text {up }}(E)>_{0}$ and $<N_{\text {dn }}(E)>_{0}$, and adopts the acceptance probability (6). In order to follow this recipe [27], one needs to determine the quoted estimates from a previous computer run. According to $[27]$, these previous estimates do not need to be very accurate. However, actual numerical tests [29] show results for $\left\langle N_{\text {up }}(E)\right\rangle$ and $\left\langle N_{\text {dn }}(E)\right\rangle$ worse than the inputs $<N_{\text {up }}(E)>_{0}$ and $<N_{\text {dn }}(E)>_{0}$ themselves! A better possibility is the random walk dynamics originally used in order to test the broad histogram method [1]. It is the same as the flat histogram, equation (6) or (9), with the approximation A1 (optionally, also A2 and A3) quoted in last section. A1 consists in taking the current, already accumulated values of the histograms $N^{\mathrm{up}}(E)$ and $N^{\mathrm{dn}}(E+\Delta E)$, in real time during the computer run itself, instead of the true averages at the right-hand side of equation (6) or (9). Results for the same tiny system treated before are shown in Table X. This approach is the same as the multicanonical real-time-measured transition probability already adopted in other earlier methods, for instance the entropic sampling [6]. A criticism to this approach is its non-strictly-Markovian, history-dependent character. According to [29], it is actually better than the fixed transition probability proposed in [27]. Approximation A2 consists in neglecting $\Delta E$ in the numerators of equation (6) or (9), breaking the symmetry between levels $E$ and $E+\Delta E$, and cannot be applied to this tiny system due to the spectrum discretness. This approach A2 is also shown to violate a particular detailed balance condition [27]. A3 consists in taking all possible values for $\Delta E$ together, by using equations (5a) and (5b). Both approximations A2 and A3 (but not A1) are bad: a) at very low energies, for any system; b) at any energy, for tiny systems.

The difference between the dynamics adopted in Tables VII to $\mathrm{X}$ and the true canonical rule adopted in Tables II to VI is the following. Canonical simulations adopt the same acceptance probability $\exp (-\Delta E / T)$ for any tossed movement increasing the energy by $\Delta E$, no matter which is the current energy $E$. As a consequence, the energies visited during the run became restricted to a narrow window around the canonical average $\langle E\rangle_{T}$. The larger the system size, the narrower 
is this window. On the other hand, within the noncanonical rules adopted in Table VII to $\mathrm{X}$, the acceptance probabilities depend also on $E$ (and $E+\Delta E$ ). As a consequence, instead of a narrow distribution of visits, one gets a broad distribution covering the whole energy axis. This feature is, of course, a big advantage over canonical rules, because only one run would be enough to cover a large temperature range. Nevertheless, the numerical results could be wrong, depending on the ac- tual dynamic rule one adopts (Table VII, for instance). A better conceptual, theoretical understanding of these and other E-dependent dynamic rules is needed, and lacking. Concerning BHM, the only constraint to be considered is the uniform probability visitation inside each energy level, separately. For other, reweighting methods based on the actual visitation profile $V(E)$, also the detailed relative distribution between different energy levels must be considered.

$\begin{array}{rcccccc}E & V(E) & \left\langle N^{\mathrm{up}}(E)\right\rangle & \left\langle N^{\mathrm{uP}}(E)\right\rangle & \left\langle N^{\mathrm{tie}}(E)\right\rangle & \left\langle N^{\mathrm{dn}}(E)\right\rangle & \left\langle N^{\mathrm{dn}}(E)\right\rangle \\ & \times 10^{8} & (\Delta E=4) & (\Delta E=2) & (\Delta E=0) & (\Delta E=2) & (\Delta E=4) \\ 8 & 4.53 & 6.1123 & 6.3407 & 1.81151 & 0.90579 & 0.82973 \\ 10 & 4.53 & 3.5553 & 7.0376 & 3.5552 & 1.55561 & 0.29630 \\ 12 & 4.53 & 2.3112 & 5.9704 & 5.5130 & 1.81807 & 0.38733 \\ 14 & 4.53 & 1.13190 & 5.5848 & 5.8873 & 2.9432 & 0.45272 \\ 16 & 4.53 & 0.75283 & 3.6920 & 7.1108 & 3.6912 & 0.75321\end{array}$

Table IX Flat histogram [27] simulation with symmetric $E$-dependent Boltzmann acceptance probability $\exp \left[-\Delta E / T_{m}(E)\right]$, equation (9), to be compared with the exact values in Table I.

$\begin{array}{rrccccc}E & V(E) & \left\langle N^{\mathrm{up}}(E)\right\rangle & \left\langle N^{\mathrm{up}}(E)\right\rangle & \left\langle N^{\mathrm{tie}}(E)\right\rangle & \left\langle N^{\mathrm{dn}}(E)\right\rangle & \left\langle N^{\mathrm{dn}}(E)\right\rangle \\ & \times 10^{8} & (\Delta E=4) & (\Delta E=2) & (\Delta E=0) & (\Delta E=2) & (\Delta E=4) \\ 8 & 3.51 & 6.1131 & 6.3399 & 1.81121 & 0.90558 & 0.83023 \\ 10 & 4.65 & 3.5553 & 7.0372 & 3.5561 & 1.55532 & 0.29619 \\ 12 & 17.03 & 2.3106 & 5.9717 & 5.5124 & 1.81788 & 0.38749 \\ 14 & 3.81 & 1.13212 & 5.5850 & 5.8865 & 2.9434 & 0.45296 \\ 16 & 2.13 & 0.75298 & 3.6917 & 7.1108 & 3.6915 & 0.75305\end{array}$

Table X Random walk dynamics [1] simulation with approximation A1 (see text), to be compared with the exact values in Table I.

Up to now, we have tested many different dynamic rules in order to measure the microcanonical averages $\left\langle N_{\text {up }}(E)\right\rangle$ and $\left\langle N_{\text {dn }}(E)\right\rangle$ from which one can determine the desired quantity $g(E)$ by BHM equation (4). The most efficient approaches are the $E$-dependent rules (Tables VIII, IX and X), where a single computer run is enough. Among them, under a practical point of view, the random walk dynamics [1] corresponding to Table $\mathrm{X}$ is the best choice, once one does not need any previous knowledge about the quantities $\left\langle N_{\text {up }}(E)\right\rangle$ and $\left\langle N_{\mathrm{dn}}(E)\right\rangle$ to be measured. This approach corresponds to approximation A1. The other two further approximations A2 and A3 [1] improve even more the efficiency. However, due to the energy spectrum discreteness, they are limited by the constraint $\Delta E<<E$ : one needs to avoid them for tiny systems, or very near the ground state even for large systems, where this constraint cannot be fulfilled. All this matter corresponds to the subject discussed in reference [27]. Let's now discuss another, subtle, further possible source of unaccuracies, which may appear when one abandon the safe canonical dynamical rules and adopts non-canonical, E-dependent dynamics in order to sample the whole energy axis during the same computer run.

In order to introduce the subject, let's resort again to canonical simulations, where some temperature value $T$ is fixed since beginning. Imagine one starts such a simulational process from a randomly chosen microstate $S$ : its energy $E_{S}$ would be in general far from the average value $<E>_{T}$, as well as many other features of this microstate which would be far from their equilibrium counterparts. By plotting the energy of each successive averaging microstate as a function of the time, one would get a curve fluctuating around an exponential decay to the final value $\langle E\rangle_{T}$ : at the end, only statistical fluctuations around this constant value remain. Thus, the very beginning of the Markovian chain of states will give wrong (biased) contributions to the 
final averages $\left\langle Q>_{T}\right.$. These are the so-called thermalization transient steps. Nevertheless, they represent no problem at all, because one can always take an enormous number of microstates after this transient bias is already over, pushing the systematic deviations to below any predetermined tolerance. Better yet, one can simply discard the contribution of these initial states, by starting the averaging procedure only after the transient is over. In the statistical physicists' jargon, one can assert that "the system is already thermalized", after these initial out-of-equilibrium, transient steps.

As quoted in [27], detailed balance is a delicate matter. Detailed balance conditions are useful only in order to ensure that the final distribution, chosen by the user, will be the correct one, for instance the Gibbs distribution for equilibrium canonical averages. However, these conditions do not ensure this final distribution will be reached within a finite time. In our case of interest, i.e. the $E$-dependent, broad-energy, non-canonical dynamics, the system never reaches canonical equilibrium inside each energy level. All these dynamic rules correspond to generalized Boltzmann factors $\exp [-\Delta E / \Theta(E)]$, where the "temperature" $\Theta(E)$ varies from one microstate to the next, along the Markovian chain. Concerning the energy, for instance, instead of an exponential decay to the canonical equilibrium situation, one gets a random walk visiting all energies, with fluctuations covering the whole energy axis all the time. The same large fluctuation behaviour holds also for other quantities, in particular the one for which the microcanonical averaging is in progress. This dynamics follows an eternal transient in what concerns the safe canonical framework. This feature may introduce systematic numerical deviations. Although those possible out-of-canonicalequilibrium problems cannot be observed in our Tables VIII, IX and X, they could be crucial for larger systems where large energy jumps in few steps become possible: features of a particular far energy region which the system recently cames from could introduce biases in the current energy averages. In this eternal-transient case, detailed balance conditions and all the related theorems give little help. To construct a good, efficient microcanonical simulator, by visiting the whole energy axis during a single computer run, seems to be a much more delicate matter. How to assure a uniform probability distribution inside each energy level also covering broad regions of the energy spectrum? This problem is open to new insight, new ideas.

Exemplifying how difficult would be to analyse the uniformity of visits within each energy level, let's take a simple example: a $L \times L$ square lattice Ising magnet ( $L>4, N=L^{2}$ spins), with $E=8$. Considering the magnetization density $m$, level $E=8$ is divided into three classes. The first one contains $g_{1}(8)=N(N-5)$ states with only two non-neighbouring spins pointing down (all other spins up, or vice-versa), corresponding to $m=1-4 / N$. The second class consists of $g_{2}(8)=12 \mathrm{~N}$ states with a 3 -site cluster of neighbouring spins pointing down, with $m=1-6 / N$. The third class has $g_{3}(8)=2 N$ states presenting a plaquette of four spins pointing down, and $m=1-8 / N$. No singlespin flip can transform a state with $E=4$ or $E=6$ into another state belonging to this third class, for instance. In order to assure a uniform distribution of visits, this lack must be compensated by other possible single-spin flips from $E=10$ and $E=12$. On the other hand, the visitation frequency to each one of these higher-energy states depends on feeding rates from higher yet energies, and so on. One must prescribe adequate transition probabilities to each such movement, taking into account all its consequences on the next, next-next step, and so on. Chessboard is an easier game. Fortunately, the first class containing $\sim N^{2}$ states dominates the counting for large lattice sizes. For $L=32$, for instance, $g_{1}(8)=1,043,456$ states are in the first class, representing $99 \%$ of the whole number $g(8)=g_{1}(8)+g_{2}(8)+g_{3}(8)=1,057,792$. Similar behaviours also occur for higher energies, giving us a solace: we remain with the hope that single-spin flips may lead to the desired uniformity for large enough systems. For a tiny $4 \times 4$ lattice, on the other hand, things go worse, once two further classes must be added to $E=8$ level: $g_{4}(8)=16$ states with a single line of spins down, corresponding to $m=1 / 2$; and $g_{5}(8)=8$ states with two neighbouring lines of spins down, with $m=0$. Then, only $g_{1}(8)=176$ states out of $g(8)=424$, i.e. $42 \%$, belong to the first class. This feature, of course, partially explains the bad results in Table VII. However, a detailed explanation for the good results obtained in Tables VIII, IX and X, following the same reasonings, is not easy.

\section{Improved Microcanonical Simulators}

While we have not yet a perfect and efficient microcanonical simulator, the advantages of the Broad Histogram method, i.e. the exact and completely general equation (4), are already in hands. The only problem is to obtain good estimates for the averages $\left\langle N^{\text {up }}(E)\right\rangle$ and $\left\langle N^{\mathrm{dn}}(E)\right\rangle$, as functions of $E$. One paliative solution is to use the available, not-yet perfect microcanonical simulators, perhaps with some repairing procedures. Even without any repair, the random walk naive approach introduced in [1] can be useful. First, observing Tables II to VI, one notes that some (large) degree of inaccuracy on the temperature can be tolerated still within very good precision on the final results. Second, comparing figures 1 and 2, one can note that larger 
systems present a plateau of almost constant temperatures covering a large energy range. This range is just the interesting one, namely the critical region where the specific heat diverges for $N \rightarrow \infty$. Third, with small variations of the temperature from one microstate to the next, within this region, the out-of-canonicalequilibrium problems should be strongly diminished.

Anyway, we can try to introduce some repairing procedures into the random walk dynamics [1] (or into any other $E$-dependent transition rate dynamics). The first idea is to equilibrate the system before measuring any averaging quantity at the current microstate. This can be acomplished by running some canonical steps just before the measuring procedure. Suppose one gets some microstate with energy $E$, during the random walk dynamics. As this microstate comes from other energy levels, submitted to acceptance probabilities others than the currently correct value $\exp [-\Delta E / T(E)]$, it is supposed to carry some undesired biases. Then, one can simply include some canonical steps with fixed temperature $T=T(E)$, in order to let the system relax to $E$-equilibrium, before taking the averages. $T(E)$ can be estimated at each step from the current, already accumulated histograms for $N^{\mathrm{up}}(E)$ and $N^{\mathrm{dn}}(E)$ (approximation A1), and the further approximations A2 and $A 3$ can also be adopted. All these tricks were already introduced in the original publication [1].

Another repairing procedure is simply to forbid large energy jumps. The simplest way to perform this task is to count a new averaging state after each singlespin-flip trial. Normally, one adopts $N$ trials, i.e. a whole lattice sweep, before counting a new averaging state, in order to avoid possible correlations along the Markovian chain of states. In our microcanonical case, however, different energy levels correspond to independent averaging processes, and one can try to abandon this precaution. Of course, this approach also saves a lot of computer time. Table XI shows the results obtained for a $32 \times 32$ lattice, where approximation A1 (real-time measurement of the transition rates) was adopted, with $10^{9}$ averaging states per energy level. The expected relative error due to finite statistics is thus $3 \times 10^{-5}$. Indeed, the observed deviations coincide with this, in spite of the figure 0.008 [27] predicted by detailed balance arguments. Even adopting the further approximations A2 (which explicitly violates detailed balance) and A3, the errors remain the same (last column). Once more, the possible source of unaccuracies has nothing to do with detailed balance dictating the relative frequency of visits between different energy levels. On the contrary, the only crucial point is the uniformity of visits inside each energy level, separately.

Perhaps the procedure of measuring averages after each new single-spin-flip trial could indeed introduce some bias, through some undesirable correlations along the Markovian chain, although this possibility is not apparent at all in Table XI. Perhaps this bias could appear only for much larger statistics. An alternative way to avoid large energy jumps still taking averages only after each whole lattice sweep is to restrict the random walk to narrow energy windows. This corresponds to the Creutz energy-bag simulator [24] combined with the random walk dynamics [1] inside each window. It was introduced in [19], and the results also show relative errors much smaller than those predicted by detailed balance arguments. The specific heat for a $32 \times 32$ square lattice is shown in figure 3 , where approximations $\mathrm{A} 1$, $\mathrm{A} 2$ and $\mathrm{A} 3$ were used. By restricting the energy to narrow windows, one is also restricting the temperature to small variations, thus forcing the system to be always near the canonical equilibrium conditions for any energy inside the current window. Once again, the best performance occurs at the critical region.

\section{VI - Conclusions}

The Broad Histogram Method (BHM) introduced in [1] allows one to determine the energy spectrum of any system, i.e. the degeneracy $g(E)$ as a function of the energy $E$, through the exact and completely general equation (4). First, one needs to adopt some reversible protocol of allowed movements in the system's space of states. Reversible means that for any allowed movement $S \rightarrow S^{\prime}$, the back movement $S^{\prime} \rightarrow S$ is also allowed. For Ising models, for instance, one can choose single-spin flips, to flip clusters of neighboring spins, etc. In fact, one could invent any protocol. For each state $S, N_{S}^{\text {up }}$ counts the number of such allowed movements for which the system's energy would be increased by a fixed amount $\Delta E$. Accordingly, $N_{S}^{\text {dn }}$ counts the number of allowed movements decreasing the energy by the same amount $\Delta E$. The energy jump $\Delta E$ is also chosen and fixed since the beginning. $\left\langle N^{u p}(E)\right\rangle$ and $\left\langle N^{\text {dn }}(E)\right\rangle$ are the microcanonical, fixed- $E$ averages for these two quantities, both functions of the energy. Then, in order to determine $g(E)$ from the BHM equation (4), one needs only to find a way, any way, to measure those microcanonical averages. 


\begin{tabular}{lcccc}
$E$ & $\ln \frac{g(E+2)}{g(E)}$ & \multicolumn{3}{c}{$\ln \frac{\left\langle N_{\mathrm{up}}(E)\right\rangle}{\left\langle N_{\mathrm{dn}}(E+2)\right\rangle}$} \\
300 & 1.749921 & 1.749897 & 1.749800 & 1.749992 \\
302 & 1.748650 & 1.748599 & 1.748496 & 1.748678 \\
304 & 1.747398 & 1.747387 & 1.747288 & 1.747365 \\
306 & 1.746164 & 1.746151 & 1.746055 & 1.746144 \\
308 & 1.744946 & 1.744960 & 1.744834 & 1.744905 \\
310 & 1.743743 & 1.743748 & 1.743638 & 1.743660 \\
312 & 1.742554 & 1.742590 & 1.742452 & 1.742438 \\
314 & 1.741375 & 1.741424 & 1.741305 & 1.741192 \\
316 & 1.740206 & 1.740295 & 1.740148 & 1.740000 \\
318 & 1.739045 & 1.739128 & 1.738995 & 1.738762 \\
320 & 1.737889 & 1.737970 & 1.737828 & 1.737596 \\
322 & 1.736737 & 1.736831 & 1.736655 & 1.736406 \\
324 & 1.735586 & 1.735680 & 1.735494 & 1.735275 \\
326 & 1.734434 & 1.734560 & 1.734342 & 1.734086 \\
328 & 1.733279 & 1.733420 & 1.733174 & 1.732904
\end{tabular}

Table XI Data for the $32 \times 32$ square lattice, near the critical region. Exact values are in the second column, and the next 2 columns correspond to different BHM runs. The random walk dynamics [1] with approximation A1 (see text) was adopted. A new averaging state is counted after each single-spin flip, instead of waiting for a whole lattice sweep. Nearly $10^{9}$ states per energy were sampled for ech run. The same numerical accuracy was obtained also by using the further approximations A2 and A3, last column, in spite of the detailed balance violation [27] between different energies, forced by A2. Within BHM, one does not need to care about the relative balance of visitations to different energies. Only a uniform sampling probability inside each energy level, separately, is important.

By following the same way adopted in measuring $<N^{\mathrm{up}}(E)>$ and $<N^{\mathrm{dn}}(E)>$, one can also obtain the microcanonical average $\langle Q(E)\rangle$ of the particular thermodynamic quantity $Q$ of interest (magnetization, density, correlations, etc). All these microcanonical averages are independent of the particular environment the system is currently interacting with. In other words, they do not depend on temperatures, equilibrium conditions, or any other thermodynamic concept: they are determined by the system's energy spectrum alone. Thus, after $g(E)$ is already determined through the BHM equation (4), once and forever, the same system can be submitted to different environment conditions, and its behaviour (equilibrium or not) can be studied resorting always to the same $g(E)$. Within the particular case of canonical equilibrium, for instance, the thermal average $<Q>_{T}$ of the quantity $Q$ can be obtained from equation (3), for any temperature $T$. If one decides to use computer simulations as the instrument measuring $\left\langle N^{\mathrm{up}}(E)\right\rangle,\left\langle N^{\mathrm{dn}}(E)\right\rangle$ and $<Q(E)>$, then only one computer run is enough to determine the whole temperature dependence, continuously, without need of repeating again the simulation for each new temperature.

Other computer simulation methods, the so-called multicanonical sampling strategies [5-7], also allow the direct determination of $g(E)$. All of them are based on the counting $V(E)$ of visits to each energy level $E$. Every time each energy level $E$ is visited by the current state $S$, along the Markovian chain, the counting is updated from the current $V(E)$ to $V(E)+1$. Within BHM, however, the E-histograms for $N^{\text {up }}$ and $N^{\text {dn }}$ are updated from the current $N^{\mathrm{up}}(E)$ and $N^{\mathrm{dn}}(E)$ to $N^{\text {up }}(E)+N_{S}^{\text {up }}$ and $N^{\text {dn }}(E)+N_{S}^{\text {dn }}$, respectively, for the same current state $S$. This corresponds to macroscopic upgrades instead of the mere counting of one more state. Thus, a much more detailed information is extracted from each averaging state withing BHM than any other method, giving rise to much more accurate results. Moreover, the larger the system size, the stronger is this advantage, due to the macroscopic character of the BHM quantities $N^{\text {up }}$ and $N^{\text {dn }}$.

Another advantage of BHM over other methods is its complete independence concerning the particular dynamic rule adopted in order to measure the microcanonical averages. Any recipe can be used, provided the correct $E$-functions $\left\langle N^{\text {up }}(E)\right\rangle,\left\langle N^{\text {dn }}(E)\right\rangle$ and $\langle Q(E)>$ were accurately determined. As the fundamental equation (4) is exact, any numerical deviation observed on the final results are due to the particular measuring recipe one chooses to adopt, not to the method itself. This is a big advantage, once one can choose a more adequate measuring instrument for each different system under study. The only require- 
ment to obtain correct microcanonical averages is a uniform probability distribution inside each energy level, separately. Detailed balance between different energy levels is irrelevant for BHM. All possible transitions between different energy levels are exactly counted by the BHM quantities $N^{\text {up }}$ and $N^{\text {dn }}$ themselves, not by the particular stochastic dynamic recipe one adopts. On the other hand, besides the uniform sampling probability inside each energy level, multicanonical methods also depend on the particular transition rates between different energies, which are tuned during the computer run in order to get a flat distribution of visits at the end. Detailed balance is fundamental for multicanonical methods. Thus, besides the acccuracy advantage commented before, any good dynamic rule for multicanonical sampling is also good for BHM, but the reverse is not true. Indeed, BHM allows the user to design his own profile of visits along the energy axis, taking for instance a better statistics near the critical region.

During the past half century, theoretical studies provided us with many recipes concerning detailed balance for Markovian processes, leading to Gibbs equilibrium distribution. These recipes show us how to design adequate transition rates between different energy levels. Unfortunately, there are no equivalent theoretical studies in what concerns adequate rules leading to a uniform probability inside each energy level. Perhaps this missing point is due to the fact that most studies were directed towards canonical distributions, where a sharp region of the energy axis is visited: after some transient steps (normally discarded from the Markovian chain) the system becomes trapped into this sharp region. Thus, the spread over this restricted region is supposed to occur naturally, giving rise to the required uniformity inside each level. At least for smooth $E_{-}$ dependent quantities $\langle Q(E)\rangle$, the quoted sharpness solves the problem. However, this is not the case if one needs to cover wide energy ranges. For both multicanonical sampling as well as BHM, this is an open problem waiting for new ideas, new insight. How to assure a uniform probability of visits inside each energy level? Some clues towards the answer were discussed, and some very efficient practical solutions were proposed in section $\mathrm{V}$.

\section{Acknowledgements}

This work was partially supported by the Brazilian agencies CAPES, CNPq and FAPERJ. I am deeply indebted to Jian-Sheng Wang for exhaustive and elucidative discussions about the subtleties inherent to this matter. These discussions were, at least, very useful in showing me my ignorance about the subject, and perhaps also showing us some possible ways to learn a little bit. I am also indebted to my collaborators Hans Herrmann, Thadeu Penna and mainly Adriano Lima, the youngest one who nevertheless becames the most expert on BHM.

\section{References}

[1] P.M.C. de Oliveira, T.J.P. Penna and H.J. Herrmann, Braz. J. Phys. 26, 677 (1996) (also in Cond-Mat 9610041).

[2] P.M.C. de Oliveira, Eur. Phys. J. B6, 111 (1998) (also in Cond-Mat 9807354).

[3] Z.W. Salzburg, J.D. Jacobson, W. Fickett and W.W. Wood, J. Chem. Phys. 30, 65 (1959); R.W. Swendsen, Physica A194, 53 (1993), and references therein.

[4] G.M. Torrie and J.P. Valleau, Chem. Phys. Lett. 28, 578 (1974); B. Bhanot, S. Black, P. Carter and S. Salvador, Phys. Lett. B183, 381 (1987); M. Karliner, S. Sharpe and Y. Chang, Nucl. Phys. B302, 204 (1988).

[5] B.A. Berg and T. Neuhaus, Phys. Lett. B267, 249 (1991); A.P. Lyubartsev, A.A. Martsinovski, S.V. Shevkunov and P.N. Vorontsov-Velyaminov, J. Chem. Phys. 96, 1776 (1992); E. Marinari and G. Parisi, Europhys. Lett. 19, 451 (1992); B.A. Berg, Int. J. Mod. Phys. C4, 249 (1993).

[6] J. Lee, Phys. Rev. Lett. 71, 211 (1993).

[7] B. Hesselbo and R.B. Stinchcombe, Phys. Rev. Lett. 74 , 2151 (1995).

[8] All real systems of interest are confined to a finite volume, thus the energy spectrum will be always discrete. Nevertheless, the method can be easily generalized also for the case of continuous energy spectra models, as in J.D. Muñoz and H.J. Herrmann, Int. J. Mod. Phys. C10, 95 (1999); Comput. Phys. Comm. 121-122, 13 (1999).

[9] J.-S. Wang, T.K. Tay and R.H. Swendsen, Phys. Rev. Lett. 82, 476 (1999).

[10] G.R. Smith and A.D. Bruce, J. Phys. A28, 6623 (1995); Phys. Rev. E53, 6530 (1996); Europhys. Lett. 39, 91 (1996).

[11] J.-S. Wang and L.W. Lee, Cond-Mat 9903224.

[12] M. Kastner, J.D. Munoz and M. Promberger, CondMat 9906097.

[13] R.H. Swendsen, J.-S. Wang, S.-T. Li, C. Genovese, B. Diggs and J.B. Kadane, Cond-Mat 9908461.

[14] J.-S. Wang, Comput. Phys. Comm. 121-122, 22 (1999); Cond-Mat 9909177.

[15] A.R. de Lima, P.M.C. de Oliveira and T.J.P. Penna, Solid State Comm. (2000) (also in Cond-Mat 9912152).

[16] Energy $E=2$ is not allowed, because one cannot arrange the spins on the square lattice in such a way that only two unsatisfied links appear. The same occurs also for $E=2 L^{2}-2$. Besides these two cases, any even value of $E$ in between 0 and $2 L^{2}$ is allowed.

[17] P.M.C. de Oliveira, T.J.P. Penna and H.J. Herrmann, Eur. Phys. J. B1, 205 (1998); P.M.C. de Oliveira, in Computer Simulation Studies in Condensed Matter Physics XI, 169, eds. D.P. Landau and H.-B. Schüttler, Springer, Heidelberg/Berlin (1998). 
[18] P.M.C. de Oliveira, Int, J. Mod. Phys. C9, 497 (1998).

[19] P.M.C. de Oliveira, Comput. Phys. Comm. 121-122, 16 (1999), presented at the APS/EPS Conference on Computational Physics, Granada, Spain (1998).

[20] H.J. Herrmann, J. Stat. Phys. 45, 145 (1986); J.G. Zabolitzky and H.J. Herrmann, J. Comp. Phys. 76, 426 (1988), and references therein.

[21] S.C. Glotzer, D. Stauffer and S. Satry, Physica A164, 1 (1990).

[22] C. Moukarzel, J. Phys. A22, 4487 (1989).

[23] M. Schulte, W. Stiefelhaben and E.S. Demme, J. Phys. A20, L1023 (1987).

[24] M. Creutz, Phys. Rev. Lett. 50, 1411 (1983).

[25] C.M. Care, J. Phys. A29, L505 (1996).

[26] B. Berg, Nature 361, 708 (1993).

[27] J.-S. Wang, Eur. Phys. J. B8, 287 (1999) (also in CondMat 9810017).

[28] B. Berg and U.H.E. Hansmann, Eur. Phys. J. B6, 395 (1998).

[29] A.R. de Lima, P.M.C. de Oliveira and T.J.P. Penna, J. Stat. Phys. (2000) (also in Cond-Mat 0002176).
[30] J.L. Lebowitz, J.K. Percus and L. Verlet, Phys. Rev. 153, 250 (1967); D.H.E. Gross, Phys. Rep. 279, 119 (1997), and references therein; D.H.E. Gross, Cond-Mat 9805391; M. Kastner, M. Promberger and A. Hüller, in Computer Simulation Studies in Condensed Matter Physics XI, eds. D.P. Landau and H.-B. Schüttler, Springer, Heidelberg/Berlin (1998); M. Promberger, M. Kastner and A. Hüller, Cond-Mat 9904265.

[31] N. Metropolis, A.W. Rosenbluth, M.N. Rosenbluth, A.H. Teller and E. Teller, J. Chem. Phys. 21, 1087 (1953).

[32] P.M.C. de Oliveira, T.J.P. Penna, S. Moss de Oliveira and R.M. Zorzenon, J. Phys. A24, 219 (1991); P.M.C. de Oliveira, Computing Boolean Statistical Models, World Scientific, Sigapore London New York, ISBN 98102-0238-5 (1991).

[33] P.D. Beale, Phys. Rev. Lett. 76, 78 (1996).

[34] Surprisingly, probably due to some implementation mistake, Table $2(\mathrm{~b})$ of reference [27] presents wrong values for the same data now correctly shown in Table IX. The reader could interpret this as a failure of the flat histogram dynamics [27], but this is not the case. 